\title{
Lane detection algorithm for an onboard camera
}

\author{
Mario Bellino, Yuri Lopez de Meneses, Peter Ryser and Jacques Jacot \\ Laboratoire de Production Microtechnique, École Polytechnique Fédérale de Lausanne, \\ 1015 Lausanne, Switzerland*
}

\begin{abstract}
After analysing the major causes of injuries and death on roads, it is understandable that one of the main goals in the automotive industry is to increase vehicle safety. The European project SPARC (Secure Propulsion using Advanced Redundant Control) is developing the next generation of trucks that will fulfil these aims.

The main technologies that will be used in the SPARC project to achieve the desiderated level of safety will be presented. In order to avoid accidents in critical situations, it is necessary to have a representation of the environment of the vehicle. Thus, several solutions using different sensors will be described and analysed.

Particularly, a division of this project aims to integrate cameras in automotive vehicles to increase security and prevent driver's mistakes. Indeed, with this vision platform it would be possible to extract the position of the lane with respect to the vehicle, and thus, help the driver to follow the optimal trajectory. A definition of lane is proposed, and a lane detection algorithm is presented. In order to improve the detection, several criteria are explained and detailed.

Regrettably, such an embedded camera is subject to the vibration of the truck, and the resulting sequence of images is difficult to analyse. Thus, we present different solutions to stabilize the images and particularly a new approach developed by the "Laboratoire de Production Microtechnique". Indeed, it was demonstrated in previous works that the presence of noise can be used, through a phenomenon called Stochastic Resonance. Thus, instead of decreasing the influence of noise in industrial applications, which has non negligible costs, it is perhaps interesting to use this phenomenon to reveal some useful information, such as for example the contour of the objects and lanes.
\end{abstract}

Keywords: lane departure warning, lane detection, sparc, stochastic retina, vibration, camera

\section{INTRODUCTION}

One of the most developed axes of research in automotive solution is passenger's safety. Indeed, 95\% of accidents are due to human behaviour and only $5 \%$ to defective vehicles ${ }^{1}$. Moreover, $80 \%$ of these accidents involve improper driving reaction, high speed and U-turn manoeuvre ${ }^{2}$. Analyses of these accident scenarios show that more than $40 \%$ of the accidents might have been avoided if the vehicle had been equipped with a warning system. This level of safety could rise to $95 \%$ if the vehicle could engage a safety driving response in critical situations. Thus, public research groups, automotive manufactures and suppliers, as well as other research institutions are developing the next generation of driver-assistant system

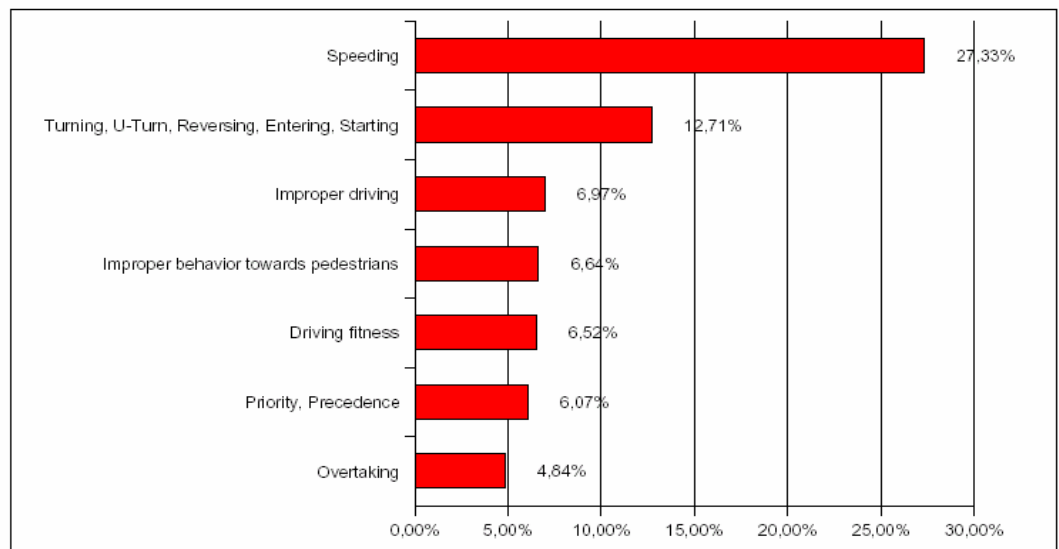

Figure 1 : "Accident scenarios" that leads to severe accidents that will enable vehicles to have safer reactions and to decrease road injuries and deaths.

\footnotetext{
* Further author information: (send correspondence to Mario Bellino)

E-mail: mario.bellino@epfl.ch, Phone: +41 216933915

Address: EPFL, STI-IPR-LPM, station 17, CH-1015 Lausanne, Switzerland
} 


\subsection{European project : Secure Propulsion using Advanced Redundant Control project}

The European FP6-STREP project "Secure Propulsion using Advanced Redundant Control" (SPARC, EU contract no.: IST-507859) has taken into account all these accidents and fixed objectives to drastically increase road safety. The main goal of the SPARC project is to improve traffic safety and efficiency for heavy goods vehicle. Trucks are particularly interesting for this project because compared to accidents with cars, heavy vehicles cause more than two times heavy damage, nearly two times more injuries and more than three times the number of persons killed. Furthermore, because of their driving distance, driving time and professional use, trucks figures out as pioneer for car technologies. To achieve SPARC aims, new technologies had to be developed and embedded.

\subsubsection{SPARC technologies}

There are two main elements in the SPARC project, the first is the X-by-wire technology that enables the steering wheel of the driver to be mechanically disconnected from the wheels. Thus, automotive industry suppresses the steering column and replaces it by wires, as in plane and aircraft technology. This equipment has been successfully tested in the Powertrain Equipped with Intelligent Technologies project (PEIT, contract no.: IST-2000-28722), and allows some automatic controls to maintain drive stability and reduce braking distance in critical situations.

The second technology is the creation of a safety assistant to compute and decide truck behaviour. This module is composed by the Human-Machine Interface $^{\dagger}$ where the driver chooses the direction and velocity to apply to the truck. This information (or stimuli) can be characterized by a vector. In parallel, the Co-Pilot technology fuses all sensor information, and provides a redundant vector which expresses the safest vehicle behaviour. Finally, the safety decision controller will generate a secure motion vector based on both previous vectors, and send this third vector to the powertrain in order to avoid accidents in case of driver failure. (See Figure 2)

Thus, the Co-Pilot has to build an internal representation of the world surrounding the vehicle in order to select the best redundant vector to achieve the safest trajectory. Although the concept is recent, it exists several projects which try to build this internal map. These "smart vehicles" are mainly implementing two separate tasks that consist in recognizing and tracking the road, or the lane limits, and in detecting and tracking other objects present in front of the vehicle, as obstacles. Several successful solutions were proposed and implemented, but all have problems with some external

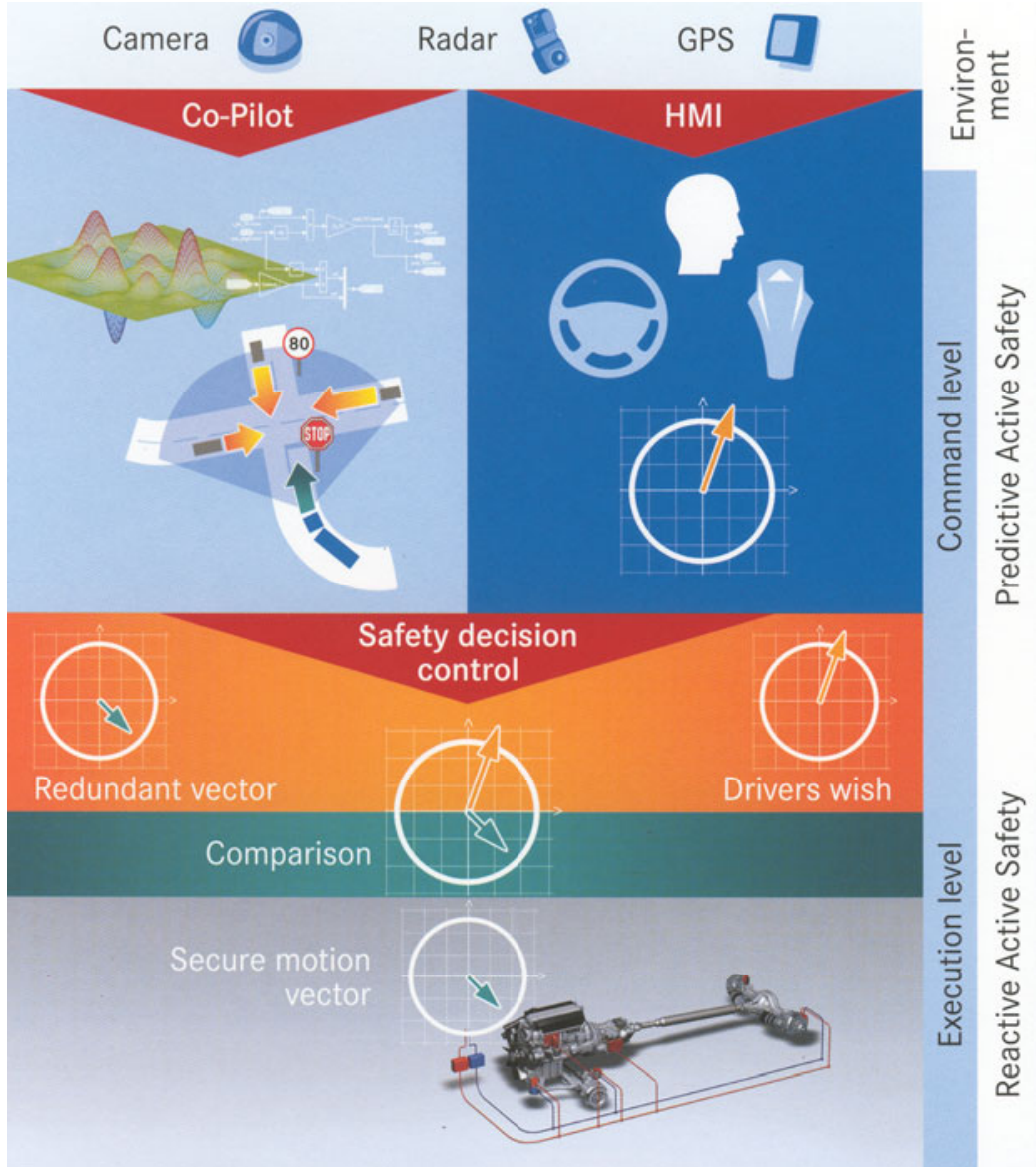

Figure 2: SPARC system using X-by-wire powertrain and assistant intelligence road specific conditions as rain, fog, shadows (or ghost), changing visibility or lighting, etc.

Our group is concerned with the development of vision-based solutions (algorithms and hardware) and consequently this paper will not deal with how to build an internal map but rather concentrate on how to find the interesting objects in the environment.

\footnotetext{
${ }^{\dagger}$ Human-Machine Interface will be denoted HMI
} 


\subsubsection{State of the art for lane detection and object recognition}

In recent development, one of the most frequently implemented solutions for road recognition is the use of camera based systems. Indeed, with the actual development of camera sensors, the cost of such devices is decreasing; furthermore, the information content of vision sensors is extremely rich. In addition, such devices have several other advantages such as, small sweep time and are passive sensors which avoid interferences with other sensors and users in the environment. Thus, the GOLD ${ }^{1}, \mathrm{ARGO}^{4}$, and $\mathrm{RALPH}^{5}$ projects were all based on such principles, using one or two cameras depending on the chosen approach. However, it is clear that such systems are mainly affected by shadows, bad weather such as fog, rain, and by changing illumination. Indeed, the contrast of the objects contained in the camera scene is strongly related to illumination condition and a variation of this value can result in the impossibility to detect the desired objects.

Other solutions can be implemented, such as laser-based ${ }^{6}$ or radar-based sensors ${ }^{7}$. These active devices are mainly used for obstacle detection but can also be used for lane detection. Their major advantage is their scanning distance (about 1$100 \mathrm{~m}$ ), the fine repeatability of measurement in dust, snow, fog and other poor weather conditions especially for radar devices. However, all projects have to deal with the resolution of such devices, sweep time, ghosts and interferences by other devices using the same physical principle.

Actually, a new kind of technology is being used. This development is based on the statement that none of the above techniques has a perfect behaviour in all situations that can occur on real road. Thus, the idea to develop a system that will fuse the data from different sensors to achieve a better result. The key idea is to use a device when it has the best result, and to replace it with another device when its reliability decreases because of external condition, as it can occur in a foggy region. Several developments have been done using some combination of the above sensors like camera$\operatorname{radar}^{8}$ or camera-laser ${ }^{9,10}$. These solutions are being further developed, because of their result in estimating real world scene with greater quality. Indeed, it is easy to imagine that the use of two, or more, sensors with a given uncertainty can result in a more precise estimation. Furthermore, in the automotive industry, the use of several sensors can yield a system that is more reliable and secure. Indeed, if a system is based on a single sensor and that it suddenly broke down, then the total system is completely unusable, which is not the case if the system uses several sensors.

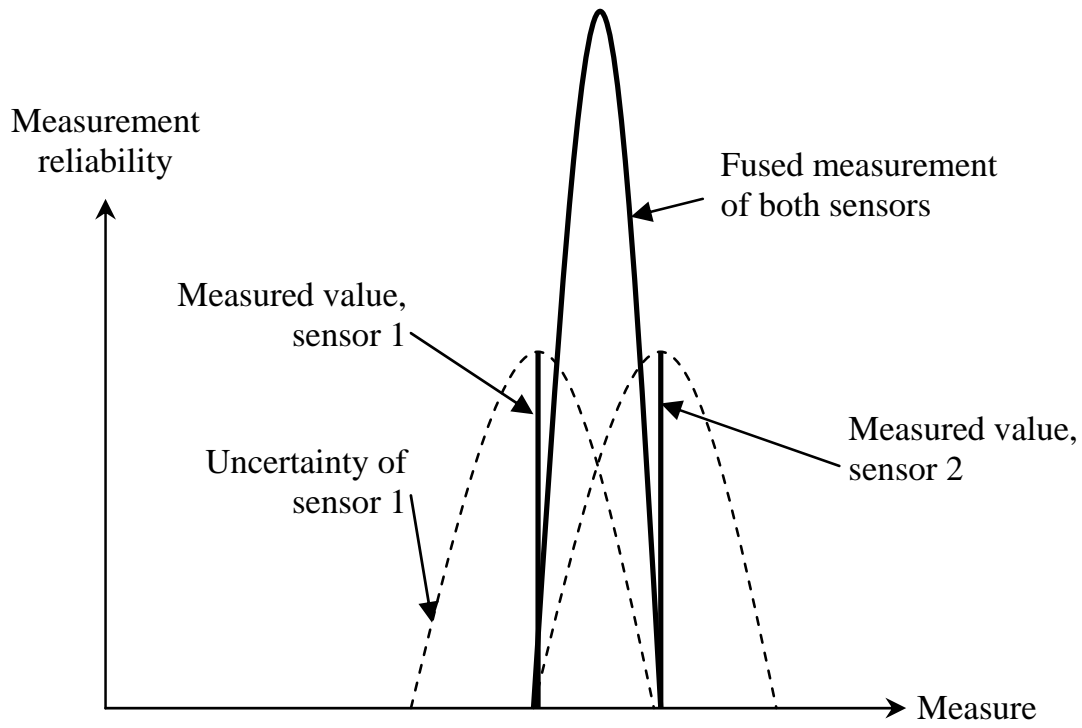

Figure 3 : Fusion of two sensors measurement increase reliability

\subsubsection{Co-Pilot technology}

Consequently, in order to build a Co-Pilot that can meet the requirement of the automotive industry and security aims of the SPARC project, the merging of three sensors has been decided. Thus, a camera, a Global Positioning System, and a radar information will be fused together to build an internal map of the environmental situation of the real world in 
which the vehicle is evolving. As detailed above the results is a much more reliable data that will aid to achieve the aims of SPARC, that are to increase security and prevent driver's mistakes by avoiding non secure behaviour of the truck.

Although, the image processing sensor is being used in collaboration with the other sensors, there will be no information exchange between the sensors. This approach allows the fusion mechanism to treat every sensor as independent, and neglect any covariance of errors.

As previously said, this article will present the current technologies that are being implemented in the SPARC vision platform. Chapter 2 will describe the lane detection algorithm that is being tested. In this chapter, an overview of the method, the problems and the possible solutions will be shown. There exists a quite infinite pool of solutions to detect the lanes of a road, however, this article will only present a possible solution, trying to find the best compromise between lane detection results and processor power. The chapter 3 will introduce the lecturer with the classical problem of embarked camera. Indeed, one of the most common problems with mobile camera is the vibration of the support system. Indeed, the roughness of the road surface, the vibration of the motor and the movement of the vehicle will induce the camera to grab a vibrating scene. Finally some conclusions will be extracted.

\section{LANE DETECTION}

The main aims of the camera are to detect the position of the truck with respect to the vehicle's lane, and perform the detection and tracking of obstacles. In order to carry out an analysis of the problem of lane detection, it is absolutely necessary to have a clear understanding of what has to be detected. Thus, the most difficult part of the algorithm is to define the hypotheses to detect the correct lane. To decrease time consumption of the algorithm, we will define that this Lane Detection Algorithm ${ }^{\ddagger}$ will work on specific and limited region of interest ${ }^{\S}$ (12 reduced ROIs are drawn in green in Figure 4). Thus, all computation algorithms are bounded to small regions and not to the whole image.

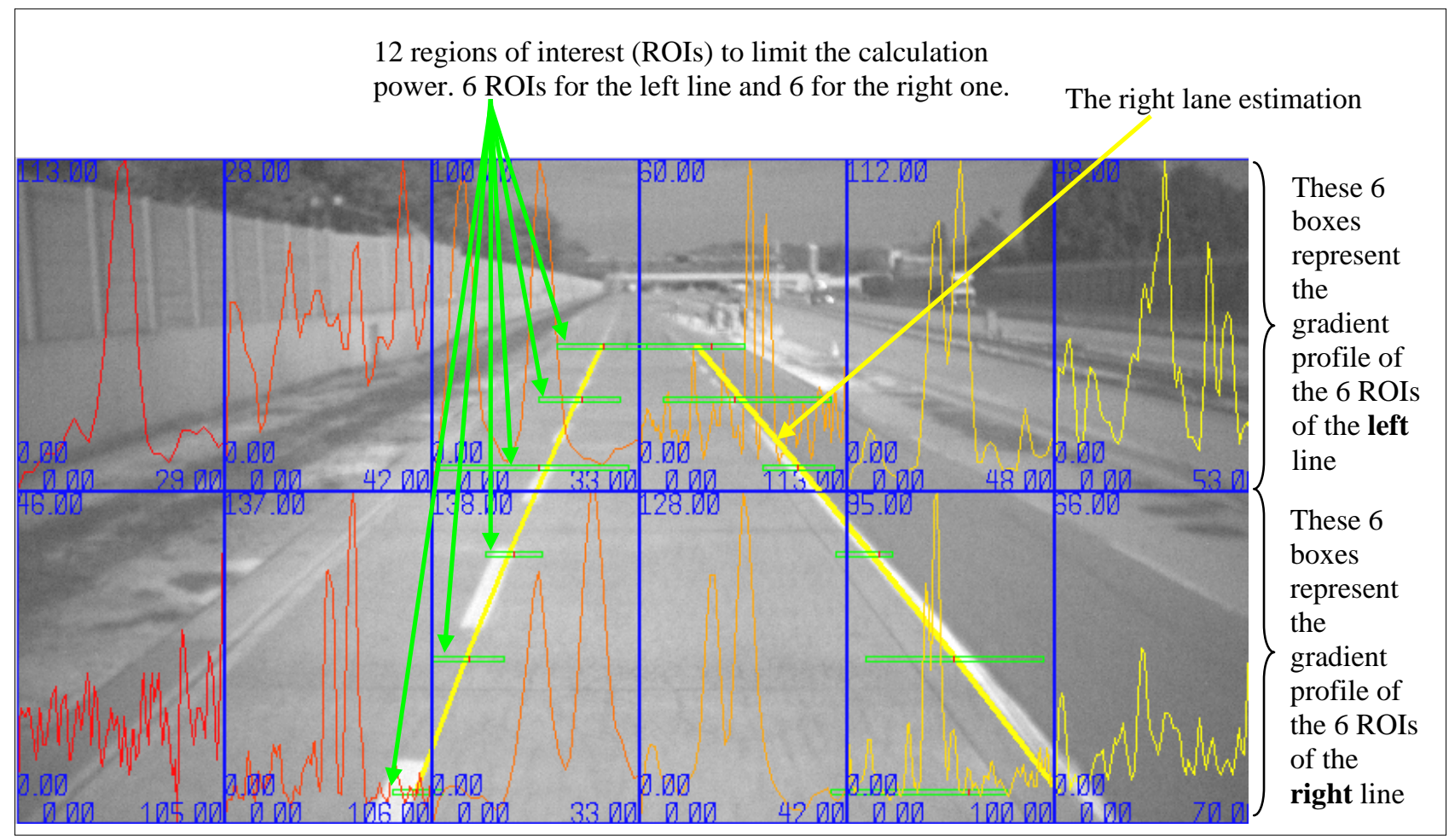

Figure 4: Example of the proposed lane detection algorithm

\footnotetext{
${ }^{\ddagger}$ Lane Detection Algorithm will be denoted LDA

${ }^{\S}$ Region Of Interest (ROI) is a term particularly used in vision computing algorithms
} 
The first assumption that is made is mainly that the road is flat, the acquisition and processing speed is close to the human fusion limit $\left(20-25 \mathrm{fps}^{* *}\right)$ and thus images are not completely uncorrelated, but evolving continuously. Furthermore, the lanes are not only defined by a clearly painted white line, but also by broken lines, and roadside limits. Consequently the pixel value, as the white painting, are not directly used, indeed, it is preferable to use the gradient of the image instead. Thus, a transition of value is sufficient to detect the roadside whatever are the delimiting objects. In order to fit the best approximation to the lane, we define by $x_{L, i}(t)$ the $x$ coordinate of the chosen point of the left lane at time $t$ in region $i$. This last variable defines the ROI and its value is null for the bottom ROI and maximum for the top one, as shown in Figure 4. Thus, a straight line, a polynomial function, or a spline, can connect all $x_{L, i}(t)$ to approximate the left line geometry. This model that will fit through every point situated on the left lane, $x_{L, i}(t)$, is denoted $P_{L}(x, y, t)$.

It is now important to describe how we will choose the best $x_{L, i}(t)$. This information is very crucial, because the performance will depend on how reliably the desired transitions are correctly found. Thus, we will implement four points of interest:

- $x_{L, i, \text { neighboor }}(t)$ is the position of gradient value that reach a minimal score, closest to the point $x_{L, i},(t-1)$.

- $X_{L, i}$, street length $(t)$ is the position of gradient value that reach a minimal score, closest to the shifted point $x_{R, i}(t-1)$. The corresponding shift is a defined value of the lane width.

- $X_{L, i \text {, straight lane }}(t)$ is the position of gradient value that reach a minimal score, closest to the ROI of a straight lane. This point will prevent the system to go completely lost, and enable the system to recover from such cases by searching the most frequent lane marker.

- $\quad x_{L, i, \text { estimated }}(t)$ is the position of estimated value of $P_{L}(x, y, t)$ in the $i^{\text {th }}$ ROI ( $i$ is related to $y$ ).

\footnotetext{
** Frames per second
} 


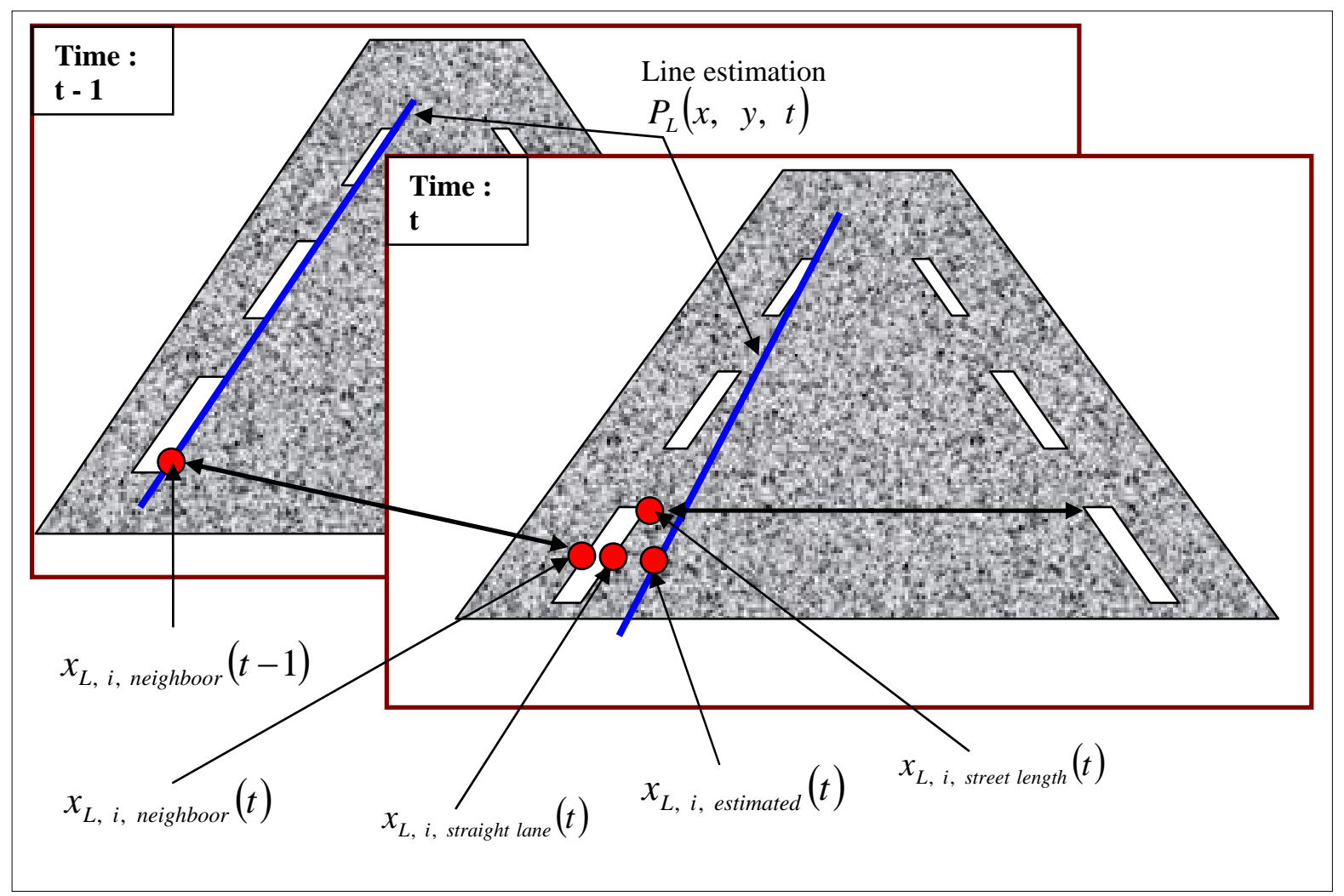

Figure 5 : Graphical interpretation of the points of interest in a single ROI

The chosen solution to estimate the parameters of $P_{L}(x, y, t)$ is to use only $x_{L, i, \text { neighboor }}(t), x_{L, i, \text { street length }}(t)$, and $x_{L, i}$, straight lane $(t)$. Then, in order to improve the result, the estimation will be re-iterate a second time with the use of all four points, and the final value of the parameters of $P$ at time $t$ will be found.

A question that can occur is to know if all four points have the same importance or do they have different weight? The next chapter will describe some possible hypothesis to characterize the points lying on roadsides, and so, attain better line detection performances.

\subsection{Hypothesis for the selection of points of interest}

As previously said, the detection of more reliable points lying along the roadsides is a critical feature, thus, we will assign a probability to each point described above. This probability describes how interesting the point seems to be, and so, how many hypothesis does the point satisfy. Indeed, a point which satisfies several criteria should have more weight than another one that fits fewer assumptions. Thus, this weighting affords a better fitting of $P_{L}(x, y, t)$ to the different $X_{L, i}(t)$. These criteria, or hypothesis, define how a lane can be expressed in a programming language:

- The relative height of the gradient of the neighbourhoods of the point, defines a hypothesis that the roadside have to contain a big contrast. Indeed, in most conventional situations the road and the side lanes have completely different pixel value.

- The distance between $x_{L, i}(t)$ and $x_{R, i}(t-1)$ is an important hypothesis. Indeed, the road or lane width is a quite known value, thus the distance between left and right lane cannot vary too much during two consecutive images. Moreover, this value must be contained in a given interval of possible road width. 
- The distance between $x_{L, i}(t)$ and $x_{L, i}(t-1)$ is also an important information. Indeed, one hypothesis is that the acquisition time between to successive images is short, thus, the distance between two successive point has to be short.

- The line width can also be found by analysing two similar peaks at a given distance (see Figure 6). If a given width is found, we are quite sure to have detected a road line.

- Line colour can also be a reliable data. However, the user must be aware that illumination changes can have severe influence on this value. Thus, a solution can be found by using a relative value, between centre of line colour and road colour.

- Distance between point and $x_{L, i \text {, estimated }}(t)$ can stick all points around a possible fitting of the roadside. Indeed, this hypothesis connects the different points in all ROI between them, and avoid that points could be completely unaligned.

- As the field of view of the camera has to deal with road perspective, the algorithm will have difficulties to found reliable data objects that are far away. As the density, in image plane, of objects situated far away is big, it increases the possibility of finding a transition that has no direct relation with the lane. Thus, the points that are close to the top of the image must have a bigger uncertainty than the bottom ones.

- Finally, after having estimated the $P_{L}(x, y, t)$ function, it is possible to compute the gradient of the image along this estimator. The relative value of this gradient along the lane estimation can help us to qualify the road boundary. (see Figure 6)

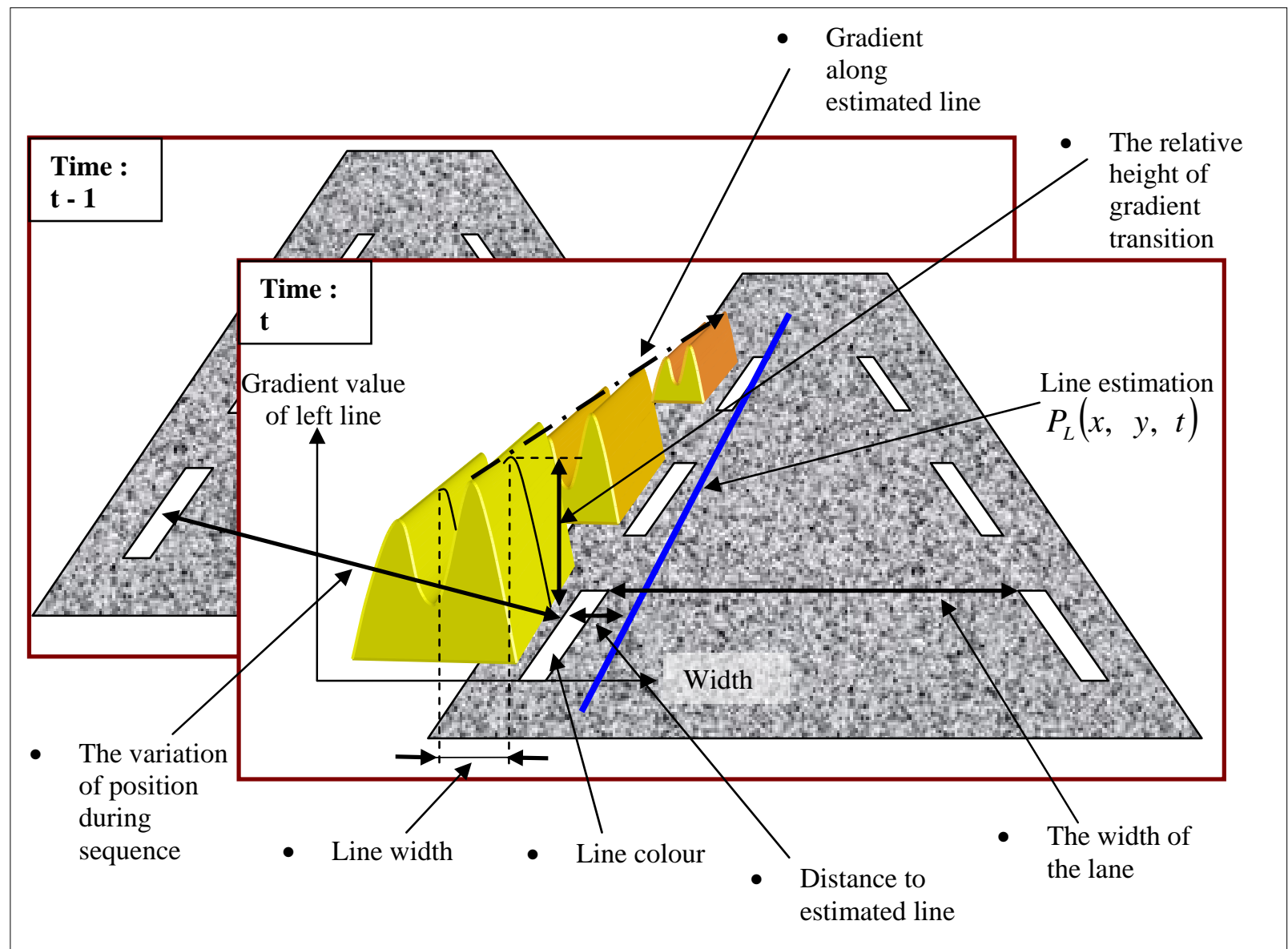

Figure 6 : Graphical interpretation of hypothesis for points of interest selection 


\section{VIBRATIONS OF EMBEDDED CAMERA}

The vibration of the embedded camera is essentially due to road asperity and motor vibrations which are widely modified by the suspension system of the truck. During the acquisition process, this motion cannot be differentiated from the motion of the objects in the camera scene. Thus, this movement can disturb several measurements, for instance, the evolution of the road curves during time sequence will be modified. Moreover, the estimation of the object velocity will be largely modified by the movement of the camera.

It is also important to consider two different motions of the camera embedded in the truck, the first one is its vibration and the second one is the pitching of the cabin due to the pneumatic system between driver's cabin and the chassis. In order to prevent the system from performing measurement errors, several solutions can be considerate.

A first solution consists in limiting the camera motion by dynamically suppressing the vibration of the vision system with an active platform. The latter must measure the motion and actuate the platform in order to maintain the system in the desired position. This solution can achieve in excellent results, but is actually unusable in automotive production because of system costs and the overall lack of robustness of a system with moving parts.

Another approach consists in implementing a model of vibration and pitching of the truck. So, by measuring several internal parameters as speed, clutch position, wheel direction and linking them to the vibration and pitching of the cabin, it is possible to build a model of the behaviour of the camera motion. This movement can finally be estimated by measuring these internal parameters, and then by applying the pre-computed model. This solution does not correct the image behaviour, but the system is now aware of the vibration condition and can, by calculation, estimate the motion of the scene's object.

Finally, the Laboratoire de Production Microtechnique ${ }^{\dagger \dagger}$ has developed another solution demonstrated in previous works $^{11}$ and 12 , in which the influence of noise can be used. This phenomenon is known as Stochastic Resonance. Thus instead of deploying several solutions to decrease the influence of noise, which have a non negligible cost, it is perhaps interesting to use this phenomenon to reveal the contour of the different objects that are contained in the image. This approach has the evident advantages to stabilize the scene but also, to freely offer an estimation of the contour. This last solution is currently being tested, and its performances are being analysed. In order to characterize the vibrations, a camera was fixed in a truck and synchronized with an inertial platform. The tests have then been done with different speeds and on different coatings of the road to evaluate the vibrations behaviour.

\section{CONCLUSIONS}

Vehicle safety is one of the most active area of research for the automotive industries. Therefore, solutions for "intelligent" vehicles are pointing out. From this approach, the European FP6 "Secure Propulsion using Advanced Redundant Control" (SPARC, EU contract no.: 507859) project is integrating such technologies to achieve safety needs. Thus, the vehicle wills integer X-by-wire technologies, which allow an autonomous co-pilot to assist the driver in critical situations.

In order to build a reliable knowledge of the environment of the vehicle, the co-pilot will fuse the information coming from several sensors. This paper describes how fusion can help to achieve the desired aims of safety of SPARC project. A specific task within the project is the extraction of pertinent information from an on-board camera. One of its main objectives is to detect the driver's lane in order to determine if driver's trajectory is the safest. Thus, a definition of "lane" has been provided, in order to determine precisely what the algorithm of lane detection is expected to find. In order to see the evolving scene, the camera and the processing platform have to be capable of taking and processing rapidly the sequence of images. A solution has been proposed to avoid large computation on the whole image, and different propositions of lane detection have been described. Several criteria had also been developed to weight the reliability of the lane detection algorithm. Finally, different solutions to reduce the influence of vibrations due to the truck movement have been proposed and will be tested in the months ahead.

\footnotetext{
†† "Laboratoire de Production Microtechnique" of the "École Polytechnique Fédérale de Lausanne" University based in Lausanne, Switzerland.
} 


\section{ACKNOWLEDGMENTS}

First of all, I would like to thanks the whole Laboratoire de Production Microtechnique, but particularly Louis-Séverin Bieri, for their help in vision algorithms. Then, thanks to all SPARC members for their materials, trucks, information and availability.

\section{REFERENCES}

1. Final Report of the European eSafety Working Group on Road Safety, http://www.eu.int/information_society/programmes/esafety/index_en.htm, 2002

2. Ansgar Maisch, Powertrain Equipped with Intelligent Technologies, an European Funded Project : PEIT, http://www.eu-peit.net/ , 2004

3. Massimo Bertozzi, Alberto Broggi, GOLD: a Parallel Real-Time Stereo Vision System for Generic Obstacle and Lane Detection, IEEE Transactions on Image Processing, vol. 7, no. 1, pp. 62-81, 1997

4. Massimo Bertozzi, Alberto Broggi, Gianni Conte, Alessandra Fascioli, Obstacle and Lane Detection on the ARGO Autonomous Vehicle, proceedings of IEEE Intelligent Transportation Systems conference, Boston, MA., 1997

5. D. Pomerleau and T. Jochem, Rapidly adapting machine vision for autonomated vehicle steering, IEEE Expert, vol. 11, pp. 19-27, 1996

6. W. S. Wijesoma, K. R. S. Kodagoda and Arjuna P. Balasuriya, Road-Boundary Detection and Tracking Using Ladar Sensing, IEEE Transactions on robotics and automation, vol. 20, no. 3, 2004

7. K. Kaliyaperumal, S. Lakshmanan and K. Kluge, An algorithm for detecting roads and obstacles in radar images, IEEE Trans. Veh. Technol., vol. 50, pp. 170-182, 2001

8. B. Ma, S. Lakshmanan, and A. O. Hero, Simultaneous detection of lane and pavement boundaries using model based multi-sensor fusion, IEEE Transactions on Intelligent Transportations Systems, vol. 1, no. 3, pp. 135-147, 2000

9. D. Dedieu, V. Cadenat and P. Soueres, Mixed camera-laser based control for mobile robot navigation, IEEE International Conference on Intelligent Robots and Systems, pp. 1081-1086, 2000

10. J. A. Castellanos, J. Neira and D. Tardos, Multisensor fusion for simultaneous localisation and map building, IEEE Transactions on Robotics and Automation, vol. 17, no. 6, pp. 908-914, 2001

11. M.-O. Hongler, Y. Lopez de Meneses, A. Beyeler and J. Jacot, The Resonant Retina : Exploiting Vibration Noise to Optimally Detect Edges in an Image, IEEE Transactions on Pattern Analysis and Machine Intelligence, vol. 25, no. 9, pp. 1051-1062, 2003

12. Mario Bellino, Stabilisation d'images par Résonance Stochastique, rapport de projet de diplôme, École Polytechnique Fédérale de Lausanne, Lausanne University, 2004.

13. Alberto Broggi, A Massively Parallel Approach to Real-Time Vision-Based Road Markings Detection, proceedings of IEEE Intelligent Vehicles Symposium, pp. 84-89, Detroit, MI, 1995

14. Karl Kluge and Sridhar Lakshmanan, A Deformable-Template Approach to Lane Detection, proceedings of IEEE Intelligent Vehicles, Detroit, MI, pp. 54-59, 1995

15. Henry Schneiderman and Marilyn Nashman, A Discriminating Feature for Vision-Based Autonomous Driving, IEEE Transaction on Robotics and Automation, vol. 10, no. 6, 1994.

16. Behzad Kamgar-Parsi, Behrooz Kamgar-Parsi and Nathan S. Netanyahu, A Nonparametric Method for Fitting a Straight Line to a Noisy Image, IEEE Transactions on Pattern Analysis and Machine Intelligence, vol. 11, no. 9, 1989.

17. Massimo Bertozzi, Alberto Broggi. Massimo Cellario, Alessandra Fasciolo, Paolo Lombardi and Marco Porta, Artificial Vision in Road Vehicles, Proceedings of the IEEE, vol. 90, no. 7, 2002.

18. Yue Wang, Dinggang Shen and Eam Khwang Teoh, Lane detection using spline model, Pattern Recognition Letters 21, pp. 677-689, 2000.

19. Yue Wang, Eam Khwang Teoh, Dinggang Shen, Lane detection and tracking using B-Snake, Image and Vision Computing 22, pp. 269-280, 2004.

20. Young Uk Yim and Se-Young Oh, Three-Feature Based Automatic Lane Detection Algorithm (TFALDA) for Autonomous Driving, IEEE Transactions on Intelligent Transportation Systems, vol. 4, no. 4, 2003. 
21. Mohsen Sharifi, Mahmoud Fathy and Maryam Tayefeh Mahmoudi, A Classified and Comparative Study of Edge Detection Algorithms, Proceedings of the International Conference on Information Technology: Coding and Computing, 2002.

22. Nicholas Apostoloff and Alexander Zelinsky, Robust Vision based Lane Tracking using Multiple Cues and Particle Filtering, proceedings of IEEE Intelligent Vehicles Symposium, pp. 558-563, 2003

23. Shih-Shinh Huang, Chung-Jen Chen, Pei-Yung Hsiao and Li-Chen Fu, On-Board Vision System for Lane Recognition and Front-Vehicle Detection to Enhance Driver's Awareness, proceedings of the IEEE International Conference on Robotics and Automation, no. 3, pp. 2456-2461, 2004. 